\title{
IMPACTOS SOCIALES DE LAS TECNOLOGÍAS DIGITALES EN EDUCACIÓN: CONDICIONANTES Y AVANCES
}

\author{
Ángel H. Facundo D., Ph.D. ${ }^{63}$
}

\begin{abstract}
RESUMEN
El presente ensayo se basa en los resultados de diversas investigaciones realizadas por el autor. Fue originalmente presentado como una conferencia que sobre el tema dictó el autor en el vil Congreso Colombiano de Informática Educativa (RIBIECOL) que se realizó en Cali, en julio de 2006. Para evidenciar los impactos sociales de las tecnologías digitales en educación, el ensayo ofrece una reflexión sobre los condicionantes, el surgimiento y evolución de la virtualidad en Colombia, el alistamiento digital del país, las características organizacionales de las instituciones educativas, avances e impactos en el sector educativo, y las tendencias. A manera de conclusión el autor señala que Colombia ha de cambiar el ciclo actual de la gestión de conocimiento. Atreverse a superar la dependencia cognoscitiva y tecnológica, produciendo aquí y ahora el conocimiento y las aplicaciones requeridas por nuestra sociedad.
\end{abstract}

Palabras claves: educación virtual, digitalidad, virtualidad, tecnologías de información y comunicación, alistamiento digital, impactos sociales.

\begin{abstract}
This essay is based on the result of diverse investigations made by the author. It was originally presented as a lecture given by the author in the VII Colombian Congress of Educational Informatics (VII Congreso Colombiano de Informática Educativa - RIBIECOL) that took place in Cali, in July 2006. In order to make clear the social impacts of the digital education technologies, the essay offers a reflection about the conditionals of the rising and evolution of the virtuality in Colombia, the digital readiness in the country, the organizational characteristics of the educational institutions, advancements and impacts in the educational sector and the tendencies. As a conclusion the author points out that Colombia must change the actual knowledge management cycle. The country must be willing to overcome the cognitive and technological dependency, producing here and now the knowledge and the applications required by our society.
\end{abstract}

Key words: virtual education, digital aspects, virtuality, communication and information technologies, digital readiness, social impacts.

Recibido: 10 de marzo de 2009

Aceptado: 3 de abril de 2009

63 Investigador/consultor. En la actualidad trabaja para la UNAD en el diseño y coordinación del Programa Doctoral en Gestión de Conocimiento. 


\section{LOS CONDICIONANTES}

Desde hace algunos años las tecnologías digitales han invadido los diversos ámbitos de la vida, por supuesto también la educación. En tales condiciones y puesto que el conocimiento es factor decisivo en el desarrollo de la sociedad actual, a la educación, y yo diría que de manera especial a las instituciones de educación superior, se les demandan una serie de logros e impactos positivos.

Ello no es siempre factible, ni su medición sencilla. De hecho, agencias especializadas como el DANE y la Agenda de Conectividad han debido desarrollar modelos de medición para determinar los avances en los diferentes sectores: productivo, educativo, estatal y en las diversas comunidades.

En el campo educativo yo diría que es una tarea, tanto operativa como conceptualmente, más compleja. En primer lugar, no se dispone siempre de información objetiva suficiente y, sobre todo, oportuna. Baste pensar que la encuesta de TIC realizada por el DANE, data del año $2001 .{ }^{64} \mathrm{Y}$, si bien existen algunas encuestas más recientes, estas no abarcan siempre la totalidad de aspectos requeridos, sino en aquellos acordes con el objetivo que persigan.

En segundo lugar, las interrelaciones entre tecnología, educación y sociedad no son lineales ni de una sola vía. Si bien es cierto que análisis históricos muestran claramente que a cada avance en las tecnologías, particularmente las de información y comunicación, corresponde un determinado tipo de pedagogía y de organización escolar, estos cambios no se dan, sin embargo, de forma espontánea ni automática. Se requieren determinadas condiciones humanas, tecnológicas, pedagógicas y organizacionales que los faciliten o los hagan factibles. Ellas se traducen en una clara conciencia sobre las características y los alcances de las tecnologías para utilizar su capacidad innovadora en beneficio de uno u otro interés. Sin embargo, ello no depende del conocimiento y decisiones individuales. Intervienen además una serie de condiciones objetivas y estrategias de acción colectiva. Pueden pasar siglos sin que los cambios que una tecnología puede propiciar, tengan lugar. Pueden ser conocidos y estar perfectamente identificados, pero verse burlados, frustrados o incluso tener efectos perversos, en dependencia de las características y condiciones sociales.

Un buen ejemplo, lo constituye el caso de la aparición del libro impreso (1451) y la tardía creación del seminario investigativo o seminario alemán como método pedagógico para su mejor utilización, así como la reorganización de funciones que propició Humboldt en la institución universitaria que lleva su nombre: la transformación del modelo de universidad tradicional en la universidad de investigación y el consecuente avance científico-técnico que desencadenó una verdadera transformación social. Con todo, esta transformación no fue universal. En muchos países, apenas si se ha avizorado. Algo semejante podría suceder con las tecnologías digitales.

64 La Encuesta de Medición de las Tecnologías de la Información y las Comunicaciones, fue realizada por el Departamento Nacional de Estadística (DANE) en junio de 2001. Abarcó 233 instituciones de educación superior, cifra correspondiente al $82 \%$ del total de instituciones del sistema. 
En tercer lugar, el desarrollo y la utilización de las tecnologías digitales son un fenómeno relativamente reciente y en desarrollo. Como tal, los referentes de medición pueden variar, puesto que la realidad es dinámica. Baste pensar que el sólo término de «educación virtual» ha evolucionado grandemente en el lapso de sólo quince años. Si inicialmente se entendía por educación virtual la aplicación de las tecnologías digitales a la «entrega» (delivery) de contenidos, hoy ello no es suficiente. En la actualidad se exige no sólo la aplicación de algunas TIC a la entrega de contenidos sino, también, al mejoramiento de los procesos de enseñanza/ aprendizaje, o mejor de aprendizaje autónomo. Más aún, desde hace unos años diversos autores europeos entienden que la virtualidad es la aplicación e integración de la digitalidad a la totalidad de las funciones educativas - la docencia, la investigación, la extensión, la administración académica一, al mejoramiento de la calidad de los aprendizajes, y, a la reorganización y transformación de la institución educativa y de la sociedad misma.

En cuarto lugar, y sólo para mencionar las complejidades más evidentes, no siempre los avances que pueden ser considerados como positivos en una determinada dirección, tienen igual valor al tomar en consideración otras dimensiones, tales como el desarrollo mismo de las tecnologías, los avances en otras áreas del conocimiento o la situación social misma.

En una palabra, no es fácil determinar impactos sociales. Tampoco es apropiado pretender aislar los impactos de una sola variable que se desenvuelve dentro de la intrincada interrelación social. Los posibles impactos sociales son el producto de un conjunto de condiciones y factores intervinientes. Cada uno de ellos puede cumplir diversos roles. Así que una valoración en una escala dicotómica positivo/negativo, no siempre es posible. Para la toma de decisiones es más útil evidenciar hechos, características y tendencias asociadas.

No obstante estas complejidades o, si se prefiere, limitaciones, en el campo de la educación superior, el IESALC (el Instituto de Investigaciones de la UNESCO sobre la Educación Superior en América Latina y el Caribe) ha venido adelantando un observatorio de la evolución de los países de la región en materia de educación superior, una de cuyas dimensiones es el uso e integración de las tecnologías digitales.

La información base para este ensayo ha sido ampliamente divulgada. ${ }^{65}$ Tuve la fortuna de participar en los estudios nacionales y regionales para sondear los condicionamientos y avances de la «educación superior virtual». Así las cosas, con base en dichos estudios y otra información que he podido recolectar posteriormente, intentaré mostrar algunos de los factores y avances que están asociados, considero, con el desarrollo social, en una u otra dirección y de forma significativa. Me referiré en particular al surgimiento y evolución de la educación virtual; al alistamiento tecnológico; a las características organizacionales; así como a las tendencias de desarrollo. Ustedes sacarán sus propias conclusiones sobre los posibles impactos.

$65 \mathrm{La}$ totalidad de los estudios nacionales y regionales se encuentran en forma digital en el portal www.iesalc. unesco.org.ve y se han publicado diversos libros con los estudios nacionales sobre educación virtual y con los estudios regionales en los diferentes aspectos de la educación superior. 


\section{SURGIMIENTO Y EVOLUCIÓN DE LA VIRTUALIDAD EN COLOMBIA}

En Colombia, y quizás de forma semejante a lo sucedido en el resto de América Latina, la virtualidad en educación (es decir, la potencialidad que se logra con el uso de las tecnologías digitales aplicadas a las diversas funciones de la educación) tiene un surgimiento y una evolución tripartita, de características peculiares.

Luego que un consorcio de trece universidades, lanzara en 1989 la denominada «Universidad Virtual» en el Instituto Tecnológico de Estudios Superiores de Monterrey (para atender las necesidades de formación del sector corporativo), diversas instituciones universitarias del país se fueron adhiriendo gradualmente, mediante convenios no siempre equitativos, a la aventura de la «virtualidad». Esta, constituye la primera línea de desarrollo. En el caso colombiano, la Universidad Autónoma de Bucaramanga fue la pionera en dichos convenios. Luego se fueron agregando las instituciones que conforman la denominada Red Mutis. Lo característico de esta línea es que dichas instituciones entran en colaboración con instituciones extranjeras o proveedores internacionales de educación, que son quienes ofrecen los programas, y que a través de dicho mecanismo comenzaron a penetrar intensamente en la región a partir de los años noventa del siglo pasado.

Una segunda línea proviene de la experimentación de núcleos de docentes en la denominada informática educativa, como forma de dar apoyo a la docencia presencial por medio de las TIC. Este ha sido un grupo que ha tenido relativa estabilidad y desarrollo sostenido. La mayoría de ustedes quienes participan en este Congreso, se ubican en esta tendencia.

Y, una tercera corriente, quizás la más tardía en surgimiento, evoluciona desde los programas e instituciones de educación superior a distancia. Tal, por ejemplo, el caso de la UNAD.

Cada línea tenía sus propias características que, de una u otra forma, influyen en el desarrollo e impactos de la virtualidad. Así, por ejemplo, por su enfoque eminentemente corporativo, la primera línea, además de una intencionalidad académica, persigue igualmente objetivos comerciales, no siempre compatibles con los requerimientos de investigación ni directamente relacionados con el desarrollo institucional autónomo, o con el logro de impactos sociales, al menos de forma prioritaria. La segunda, se dio generalmente en las instituciones presenciales más consolidadas que, en buena medida, fueron renuentes a la modalidad de educación abierta y a distancia, al menos durante la etapa inicial de surgimiento de esta modalidad en el país. Y la tercera, se vio limitada, al menos inicialmente, por las características que tenía la educación a distancia en ese momento en el país: se encontraba en la primera generación, caracterizada básicamente por diseños curriculares lineales, el ofrecimiento de módulos escritos en papel y la entrega de materiales por correo postal. El uso de multimedia análogos apenas comenzaba a generalizarse.

Y, quizás las características comunes más sobresalientes eran la escasa intercomunicación entre los grupos que trabajaban en una y otra línea; la escasa investigación, conocimientos y experiencias en materia de diseño curricular, elaboración de materiales y pedagogías de aprendizaje autónomo, de gran importancia teórica y práctica para el desarrollo de la virtualidad, que si bien podrían 
considerarse un elemento común a todas, era más evidente en las dos primeras líneas. Diversos autores, entre ellos Otto Peters, consideran que es en la modalidad de educación a distancia, donde se encontraban y encuentran las mejores condiciones para afrontar la virtualidad.

Estas y otras características menores, propias de los orígenes de la aplicación de las tecnologías digitales en educación, jugaron lo que puede denominarse como un factor condicionante que podría explicar, en no poca medida, el relativamente lento avance del país en materia de uso, extensión, experimentación e investigación sobre las tecnologías digitales en educación y sus esperados impactos sociales. No se disponía de un terreno suficientemente abonado. Al menos esto se muestra con mayor claridad durante los primeros años de evolución. Otros países contaron con condiciones más favorables y, en consecuencia, tuvieron inicios más dinámicos y de impacto.

La Red Iberoamericana de Informática Educativa ha sido al respecto un verdadero catalizador del desarrollo, en tanto que sus funciones son precisamente crear redes y nodos para compartir e intercambiar conocimientos y experiencias para avanzar conjuntamente en materia de investigación y desarrollo. Este, dicho sea de paso, es el gran mérito y la oportunidad que tiene RibieCol en nuestro país.

\section{EL ALISTAMIENTO DIGITAL DEL PAÍS}

El escaso alistamiento digital existente (en inglés, e-readiness) es otro importante factor que se debe considerar en el momento de evaluar los avances e impactos, ya que la infraestructura tecnológica, el ancho de banda, los costos de la conectividad, el escaso número de equipos de cómputo o la todavía insuficiente alfabetización digital en amplios sectores de la población, ha obrado hasta el presente como un verdadero obstáculo para alcanzar un mayor desarrollo de la virtualidad. Aunque, por fortuna, en los últimos años se han venido realizando importantes avances al respecto.

Como ustedes conocen, el alistamiento digital consiste en una serie de índices que buscan medir, a partir de conjuntos de indicadores diversos y con base en información objetiva y agregada, cuál es el nivel de preparación de un país en materia de infraestructura de conectividad, de entrenamiento, de regulación institucional, de uso de las tecnologías digitales u otras variables semejantes.

Existen diversos tipos de índices: El Índice de Acceso Digital (DAI) elaborado por la Unión Internacional de Telecomunicaciones; el índice e-Readiness o InfoDev, producido por el Banco Mundial; el Índice marco de alistamiento en red del Foro Económico Mundial (WEF) elaborado con base en tres componentes: el ambiente, alistamiento y el uso; el de la APEC que se centra en gobierno y comercio electrónico; el MOSAIC que mide la difusión global de internet; la McConnell que se centra en gobierno e infraestructura; la Harvard-cDI que analiza tecnología y participación de la comunidad; el cspp que mide comunidad y comercio electrónico; el wITSA que se enfoca en medir fuerza laboral, negocios, consumidores; el Economist Intelligence Unit que determina ambiente para negocios en red, y otros más. 
Los resultados que arrojan estos índices son indicativos. Si se toma, por ejemplo en consideración el índice DAI, los países latinoamericanos y del Caribe alcanzan tan sólo promedio de 0.46 . Es decir, se encuentran por debajo de la media, aunque podría decirse que buena parte de ellos, en virtud de la globalización, iniciaron los procesos de digitalización de forma relativamente simultánea con países de otras regiones. Colombia aparece en todos los índices con un nivel de desarrollo medio. En el índice DAI, por ejemplo, es superada no sólo por países desarrollados, sino por otros países de la región, tanto del Caribe, Centro y Suramérica, algunos considerados incluso como de menor desarrollo relativo (ver Gráfico 1). Estos datos señalan que, al menos durante los primeros años de aplicación de la digitalidad a la educación, la velocidad en el alistamiento de las condiciones para su introducción fue (y ha sido muy lenta) y, por ello, los rezagos relativos.

Gráfico 1. Índice de acceso digital (DAI). América Latina y el Caribe en comparación con Suecia, el país de mayor (DAI)

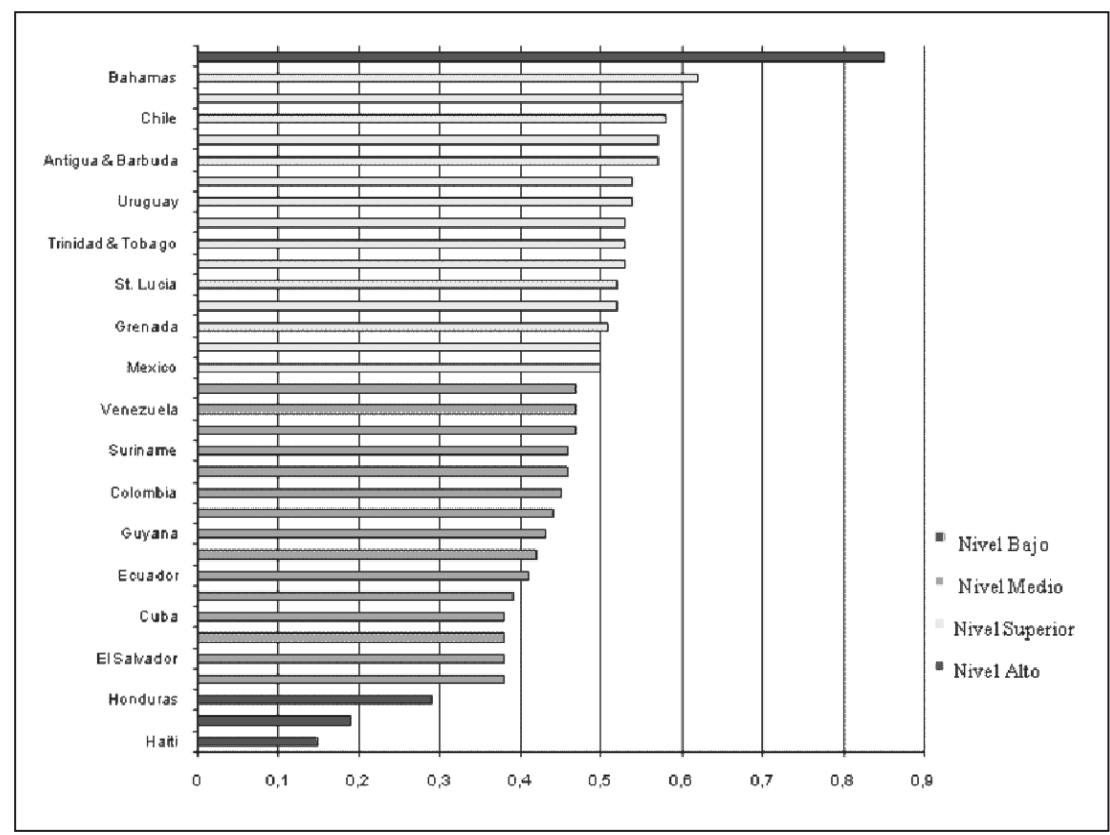

Fuente: ITU, Telecommunication Development Report, 2003.

Sin pretender aburrirlos y cansarlos con cifras (ver Anexo A. Cuadro 2) existen algunos datos que llaman la atención en el índice DAI. Las mayores diferencias con los países mejor clasificados en cada una de las variables que conforman el índice, se encuentran en el ancho de banda y en los costos relativamente alto de la conectividad. Estos serían factores objetivos que impiden una mayor introducción, calidad de uso e impactos de las tecnologías digitales. Y, si bien existen grandes brechas en las economías de los países, la diferencia no es tan marcada en la capacidad de compra de la tecnología (affordability), lo que implicaría que más que un problema 
de dinero como tal, se trataría más bien de un problema relacionado con las decisiones políticas. Este aparece sin lugar a dudas como un factor fundamental para el logro de impactos sociales.

En Latinoamérica (y, por supuesto, también en Colombia) los costos de conectividad son todavía demasiado elevados. De acuerdo con los datos DAI para el 2003, eran 145 veces más onerosos que en Hong Kong (el país con las tarifas cuya relación Internet/PIB son más favorables), 58 veces más onerosos que en Estados Unidos y 41 veces más que en Dinamarca, Taiwán, Canadá, Suiza y Alemania, que son los países más avanzados en esta materia.

El ancho de banda, que tiene que ver con la posibilidad de usar multimedia, intercomunicación e hipertextos, características centrales de la WEB 2, así como la velocidad de conexión, han sido por el contrario muy bajos. Bahamas que es el país de la región que aparece mejor clasificado, se encontraba en el 2003 en una situación 44 veces inferior a la de Dinamarca, el país mejor clasificado en materia de conectividad. La diferencia del país líder respecto a Brasil es de 377 veces, con Colombia de 1598 veces y con Haití, la diferencia es de 4830 veces. De hecho, según datos del DANE el $75 \%$ de las conexiones se hace vía telefónica, con excepción del sector de educación superior, donde el 41,9\% de las instituciones indicaron hacerlo por otros medios más veloces (ADSL, cable u otros).

De otra parte, estadísticas recientes señalan que el servicio de banda ancha en la región está concentrado en Brasil (49.6\%), seguido por México (13.9\%), Argentina (11.9\%), Chile (10.7\%), Perú (5.6\%) y Venezuela (4\%). Colombia aparece tan sólo con un 3\%. El resto de países representan el 1.4\% (ver Cuadro 3). La mayor dinámica de penetración de la banda ancha durante el último año se está dando en Chile (14.6\%), Argentina (6.2\%), Brasil (5.4\%) y Perú (5.45\%).

Gráfico 2. Distribución de la banda ancha en América Latina

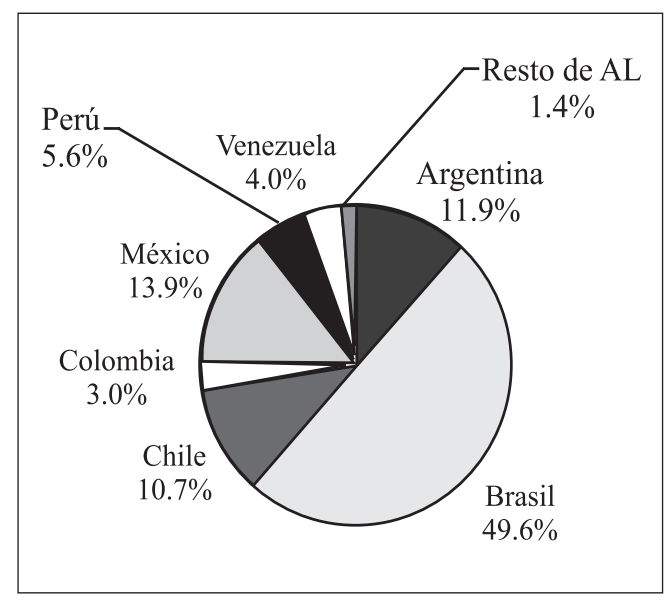


Costos elevados y un ancho de banda reducido, tienen una gran incidencia en la aplicación y usos más efectivos de las tecnologías digitales en educación. Y son factores intervinientes por fuera del ámbito de acción del sector educativo propiamente dicho.

Nuestro país parece haber comprendido, relativamente tarde, la necesidad de superar esta situación, en buena medida debido al monopolio de operadores en el sector de telecomunicaciones. Sin embargo, las consecuencias sociales y económicas de los rezagos, la presión de sectores académicos y el ingreso del sector privado externo con mayor capacidad de innovación en el manejo de las telecomunicaciones nacionales, han hecho que los servicios de banda ancha se incrementen en los últimos años. Igualmente, se han introducido importantes reducciones en las tarifas de internet.

Por ejemplo, entre el 2000 y el 2005 la conexión por cable experimentó en el país una variación del $470 \%$, de acuerdo con el informe sectorial de la Comisión de Regulación de las Telecomunicaciones (CRT) del año 2005. Según datos de una encuesta reciente del Centro Nacional de Consultoría, el tipo de conexión ha variado en el país en tan sólo dos años: 20\% de los hogares usa en la actualidad xDSL; 29\% cable; y el servicio conmutado análogo se ha reducido al 51\%. Las conexiones inalámbricas, generalmente con tecnología WiMax, se han venido igualmente extendiendo, particularmente en ciudades como Bucaramanga y Bogotá, durante los últimos años. Sin embargo, el aumento de velocidad, es todavía bajo. De acuerdo con una encuesta del Centro Nacional de Consultoría, en el 2005 sólo el 19\% de los usuarios con este servicio dispone de velocidades entre 200 y $600 \mathrm{Kbps}$.

La situación en materia de infraestructura informática es igualmente deficitaria y desigual. Según datos del DANE sólo el 18.2\% de los hogares colombianos disponía de computadores en el año 2003. Y, de ellos sólo el 44.7\% tenían conexión a internet. La encuesta citada del Centro Nacional de Consultoría estaría indicando que esta última cifra habría subido al $54.2 \%$ en el 2005. Es decir, en promedio uno de cada dos hogares. Sin embargo, se muestra todavía una clara diferenciación por estratos socioeconómicos: $37 \%$ en el estrato uno; $42 \%$ en el dos; $53 \%$ en el tres; $73 \%$ en el cuatro; $80 \%$ en el cinco y $78 \%$ en el seis. Y el mayor volumen de usuarios lo constituyen los estudiantes de bachillerato y educación superior.

La distribución regional es así mismo desigual. Según el DANE, el 81.9\% de los computadores disponibles en los hogares se encontraban altamente concentrados en las tres ciudades principales: Bogotá (55.9\%), Medellín (14.9\%) y Cali (11.1\%). Y en el sector de educación superior en cinco ciudades. El 51\% en Bogotá, el 16\% en Antioquia, el 9\% en Valle, el 5\% en Atlántico y el 5\% en Santander. Y, si bien programas como COMPARTEL y Computadores para Educar han sido importantes, estos no logran corregir las tendencias antes indicadas. Estos datos estarían indicando que los impactos no sólo son diferenciales, sino inequitativos.

\section{LAS CARACTERÍSTICAS ORGANIZACIONALES DE LAS INSTITUCIONES EDUCATIVAS}

Una mirada a la situación organizativa de las instituciones de educación superior con programas virtuales, muestra igualmente características que inciden en los impactos sociales. 
En una encuesta realizada por el autor en el año $2003,{ }^{66}$ se pudo identificar que si bien los programas de educación virtual en el país son realizados por los diferentes tipos de instituciones que conforman el sistema de educación superior, el mayor porcentaje se concentra en las instituciones universitarias $(52 \%)$ e instituciones tecnológicas ( $8 \%$ ). De acuerdo con las respuestas, el objetivo básico que se persigue es el incremento de la matrícula mediante el ofrecimiento de «programas a distancia/ virtual». La mayor concentración de la virtualidad se da en instituciones privadas (72\%). Con todo, ellas utilizan las TIC en programas de educación presencial, pero sobre todo en educación a distancia (96\% de los casos). El tipo de organización institucional más usado (52\% de los casos) es el de equipos «mixtos» de docentes que simultáneamente atiende programas presenciales y a distancia. Este hecho es un indicador de la superación gradual del viejo enfrentamiento entre las dos modalidades, gracias a la virtualidad. Con todo, el número de docentes trabajando en equipos para el desarrollo de la virtualidad es reducido. Sólo una institución contaba con un equipo de más de cien docentes dedicados al tema. Cerca de la mitad de las instituciones (48\%) contaba con equipos integrados por menos de diez docentes; $28 \%$ contaba con equipos entre once y treinta docentes; y $12 \%$ con equipos entre treinta y uno y cien docentes. La mayoría de las instituciones no disponían de tradición sólida en materia de aprendizaje autónomo y distribuido. La matrícula presencial y las formas tradicionales de enseñanza continúan predominando ampliamente, incluso al utilizar medios digitales. De igual forma, existe un claro predominio del modelo organizativo tradicional, en el cual prima la función de docencia. Finalmente, según la encuesta, existe una marcada ausencia de políticas nacionales e institucionales para la innovación en materia de modelos pedagógicos y organizacionales más acordes con el uso de las TIC.

Todos son igualmente factores que indican los impactos sociales que pueda estar teniendo en el país el uso de las tecnologías digitales.

\section{AVANCES E IMPACTOS EN EL SECTOR EDUCATIVO}

Comparado con otros sectores y dentro de la situación antes descrita, el sector educativo colombiano presenta, con todo, avances importantes, particularmente durante los últimos años. Y, si bien los tiempos de operación y maduración de la mayoría de los programas son todavía demasiado cortos, el impacto general de algunos de estos logros, es ya perceptible estadísticamente.

Tal es el caso de la UNAD, institución que ha dado un salto de la fase de educación a distancia «postal» (fase I) a la «digital» (fase III). Como consecuencia, su matrícula es una de las mayores a nivel de la educación superior del país. Igualmente se han venido consolidando otras instituciones y programas, particularmente aquellos más antiguos. Otras medidas, de corte más cualitativo, están produciendo cambios tanto en la vida de las instituciones, en el sistema educativo y, a nivel de la sociedad. Resaltaré tan sólo unos pocos, los que considero más relevantes.

66 La Encuesta sobre la Educación Superior Virtual en Colombia, se llevó a cabo durante el segundo semestre de 2002 y los primeros días de 2003. Fue la base sobre la cual se realizó el trabajo sobre la Educación superior virtual en Colombia. 
El primero y más evidente, a juzgar por los índices de alistamiento digital, es la capacitación relativamente masiva que se ha dado en el país en materia de conocimiento de las tecnologías digitales de información y comunicación. Son múltiples los programas oficiales y privados que han contribuido a este logro. En términos generales, las tecnologías digitales, en particular el uso del computador y de internet, sin ser los más generalizados (el teléfono móvil es sin duda el de mayor crecimiento e impacto) ha venido cambiando la actividad y la vida de los colombianos. Por supuesto que aún persisten tanto las brechas digitales como las desigualdades socioeconómicas que matizan las afirmaciones, frente a los promedios. Y, al compararnos con otros países de la región, los rezagos son igualmente evidentes. Considero que esta situación es suficientemente conocida por todos, por lo que no me detengo en ella.

Un segundo avance, para mí de gran significación, se da en la convergencia que se ha venido dando entre las tres líneas de desarrollo antes mencionadas. Si hasta hace pocos años era notorio el distanciamiento y el desconocimiento mutuo entre ellas, hoy esta situación parece haberse comenzado a superar en forma definitiva. Este no ha sido un proceso fácil. Sin duda, fue favorecido más por la convergencia propia de las tecnologías digitales (de hecho, yo la considero como característica fundamental de las TIC), que por políticas explícitas. Baste pensar que, si todas las anteriores tecnologías buscaron sustituir en buena medida a sus antecesoras, las TIC digitales han propiciado, por el contrario, la integración de todas las tecnologías precedentes. Sin duda, las que ofrecen mayores posibilidades son las tecnologías que permiten interactuar en redes cada vez más amplias, en tiempo real, de forma gratuita o a muy bajos costos: el Wiki no sólo para interactuar sino para la producción colectiva de textos; la multimedialidad y el hipertexto que ofrece amplias posibilidades de flexibilidad, en materia de educación.

La convergencia de tecnologías, propia de digitalidad, ha facilitado el diálogo, el necesario conocimiento mutuo y el acercamiento entre ingenierías y pedagogía; entre los diferentes lenguajes (escrito, gráfico, imagológico, pedagógico e informático que ellas involucran), al igual que entre las epistemologías y métodos de trabajo. Esta convergencia está permitiendo al mismo tiempo avanzar igualmente en la superación de las oposiciones y barreras artificiales entre las diferentes ciencias; entre la presencialidad y la distancia (oposición cada vez más ficticia); o entre cantidad y calidad, tan típicas de la etapa inicial de aplicación de las tecnologías digitales a la educación. Al mismo tiempo, fomenta objetivamente la integración y coordinación de esfuerzos investigativos entre las diversas líneas con las cuales se inició en el país la denominada «educación virtual».

La tendencia convergente se evidencia así mismo, en la consolidación de equipos interdisciplinarios al interior de las instituciones, también en un gradual proceso de transición de proyectos de investigación exploratoria y diagnóstica (propias de los primeros momentos), para comenzar a adentrarse en experimentación y desarrollos más avanzados. Una consulta a la base ScienTi de Colciencias, permite apreciar este proceso que yo llamaría de maduración. De una parte existe una importante masa crítica en materia de exploración. En el área de TIC se reportan 13236 proyectos en el 2006. De otra parte, evidencia una clara transición de las temáticas a través del tiempo: del diagnóstico y caracterización del uso de las TIC en 
las diferentes regiones geográficas, disciplinas y niveles educativos, se han venido abriendo paso proyectos que tienen por objetivo la búsqueda de nuevos horizontes en materia de aprendizajes e incluso de desarrollos tecnológicos específicos.

Si bien estos avances son muy prometedores, todavía es demasiado pronto para encontrar resultados e impactos positivos. La investigación y sobre todo el desarrollo tecnológico, insisto, requieren procesos de maduración. Sin embargo, al compararnos con otros países se evidencian rezagos, sin duda, condicionados, al menos en parte, por el escaso alistamiento digital del país en materia de conectividad. Con todo, avances que aún dentro de tales condiciones podrían ser posibles, no parecen ser, cuando menos, relevantes. Tal sería el caso, por ejemplo, en materia de mejoramiento de los aprendizajes con ayuda de las TIC. Así, en materia de contenidos digitales o «virtuales», todavía se privilegia el texto, en relación al empleo de medios audiovisuales, y la interactividad y la simulación apenas si se utilizan. En el SENA y otras pocas instituciones tanto de educación básica como de educación superior, se aprecian algunos avances al respecto, utilizando el blended learning o combinación entre simulación y presencialidad. Sin embargo, buena parte de estos logros han sido posibles gracias a la importación de software que bien podría haber sido desarrollado en el país. En una palabra, a juzgar por los proyectos que se presentan a Colciencias, no pareciera haber mayor interés investigativo al respecto. ¿O, quizás, sea la escasa financiación o incentivos lo que explica este fenómeno?

De otro lado, los curricula virtuales son todavía lineales e inflexibles, y no se evidencian experiencias e investigaciones significativas trabajando sobre este problema. Es decir, aún dentro de las condiciones existentes en el país, no se estarían aprovechando los medios digitales disponibles, y que perfectamente podrían ser utilizados de forma asíncrona sin tener que recurrir a servicios en línea. La experiencia de otras instituciones y países es indicativa al respecto. Tal es el caso de la Universidad Nacional Abierta de Corea del Sur que ha desarrollado cerca del $80 \%$ de sus contenidos en forma audiovisual y los entrega sin recurrir al uso del internet, no obstante ser su conectividad mucho más avanzada que en nuestro país. Con ello podrían tenerse desarrollos más congruentes con el alistamiento digital y con los resultados científicos en materia de los múltiples tipos de inteligencia humana, así como del predominio de la cultura oral y audiovisual de nuestra población y el escaso desarrollo de competencias de lecto-escritura.

Quiero llamar la atención sobre estos aspectos, pues si bien en las primeras etapas de desarrollo de la digitalidad se la ha asociado con la «entrega» sincrónica de contenidos, no es la única opción, ni siempre la más apropiada. Algo semejante podría afirmarse en relación con la superación de los modelos pedagógicos tradicionales, hay que recurrir y explorar la multimedia, el hipertexto y la simulación como forma de flexibilizar los programas, o recurrir a una mayor indagación, experimentación e investigación como fundamentos de los procesos cognitivos. Las tecnologías digitales ofrecen amplias posibilidades al respecto, no necesariamente supeditadas a los condicionamientos derivados de la escasa conectividad. Quizás factores ligados a las características de los grupos pioneros en materia de virtualidad podrían estar asociados a estos fenómenos. El país debiera emprender análisis más profundos 
sobre estos aspectos e incrementar los incentivos en materia de producción de objetos virtuales de aprendizaje, donde podríamos tener ventajas comparativas, aún dentro de la actual situación de alistamiento digital.

Otros factores podrían estar igualmente asociados: nos falta una exploración sistemática sobre la semiología, conocer otros signos, códigos y lenguajes. Una opción práctica al respecto podría ser, por ejemplo, integrar a productores de teatro, cine y diseño gráfico a los equipos interdisciplinarios de desarrollo de la virtualidad. Estos son sectores profesionales que cuentan con desarrollos importantes en nuestro medio, y podrían hacer grandes aportes al desarrollo de la digitalidad aplicada a la educación.

Otro campo, que parece haber perdido la dinámica que alcanzó en años anteriores es el de desarrollo de software educativo. Por supuesto que este exige mayores conocimientos y recursos. Entre ellos, contar con núcleos estables, competitivos y bien remunerados a escala internacional. Desde ningún punto de vista es conveniente que el país decaiga en esta área o que sus mejores investigadores tengan que emigrar. Uno de los tantos esfuerzos en que debe incursionarse en el campo educativo es en el desarrollo de plataformas de administración de aprendizajes más acordes con las posibilidades que ofrece la wEB 2 en materia de aprendizajes. Si bien en teoría es un lugar común hablar de modelos pedagógicos flexibles, estos encuentran no pocos obstáculos en las características de los LMS disponibles. De otra parte, el aprendizaje autónomo y distribuido parece haberse limitado a la superación de las barreras de tiempo y espacio en la recepción de unos contenidos que se deben interiorizar. Es indispensable que el país continúe experimentando en el desarrollo de pedagogías, métodos y software especializado que, en la práctica, fomenten y se basen cada vez más en la búsqueda e indagación de información, en la investigación y en la construcción y estructuración de conocimientos. Pareciera que, en una u otra materia, la competencia desigual frente a las plataformas comerciales hubiera desincentivado el interés investigativo nacional al respecto. Existen muchos otros campos que ustedes conocen mejor que yo, y que sin duda serán debatidos en este Congreso.

Un tercer avance significativo se evidencia en materia del uso e integración de las tecnologías a las diferentes funciones educativas. Insisto en que el concepto contemporáneo de «educación virtual» se relaciona con la totalidad de funciones educativas y no sólo con la docencia/aprendizaje. Si se analizan los portales existentes, numerosas instituciones han pasado de la etapa informativa inicial al ofrecimiento de múltiples servicios en línea para sus usuarios. Tal es el caso de muchos portales universitarios, igualmente, el caso de programas desarrollados por el Ministerio de Educación, tanto a nivel de educación básica (como el portal Colombia Aprende), o el caso de portales desarrollados por iniciativa de las ONG o grupos privados nacionales, como el portal Eduteka que presta amplios servicios a educadores y estudiantes, para nombrar tan sólo uno de los más sobresalientes.

Quiero hacer mención especial del nuevo sistema SNIES (Sistema Nacional de Información en Educación Superior) y del programa SPADIES para detectar y pronosticar la deserción, recientemente elaborados para el MEN, por talento colombiano. Uno y otro son desarrollos nacionales de software que enlaza en línea diversas bases de datos existentes en instituciones 
del sector (MEN, COLCIENCIAS, ICETEX y otras) con las bases de datos estandarizadas de las propias instituciones de educación superior. Por medio del primero se busca incorporar, de forma automatizada, la información para hacer más transparente la información a la comunidad. El segundo, hace seguimiento individualizado a cada estudiante y busca prevenir con sistemas de alerta temprana, el flagelo de la alta deserción estudiantil. Con ellos el MEN ha realizado un salto cualitativo importante y tomado ventaja en los programas de informatización del sector gobierno. También se cuenta con el programa sACES para los trámites de registro calificado de programas educativos. Tengo noticias que se desarrollan programas que posibiliten en línea los diversos trámites institucionales: certificado judicial, antecedentes disciplinarios, otros. Empero, aún faltan muchos otros programas tanto en el sector educativo como en sectores relacionados con él.

Un cuarto y último avance digno de mencionar es el esfuerzo por consolidar las redes académicas regionales como Rumbo, Ruana, Rumba y otras más, que han sido el paso previo para la creación de la Red RENATA como autopista de alta velocidad que permitirá compartir el uso de programas disponibles en red, logrando importantes ahorros a las instituciones pero, sobre todo, conectar a las instituciones académicas y al país con las redes académicas regionales (como CLARA) y con las redes internacionales. Todos estos avances y muchos otros que no he mencionado, significan impactos positivos, al mismo tiempo que inequidades, de no corregirse las concentraciones y exclusiones actuales. Por esa razón, acelerar la Agenda de conectividad es sin lugar a dudas uno de los factores generadores de mayores impactos.

\section{LAS TENDENCIAS}

Si bien no es fácil determinar los impactos sociales, es aún más complejo intentar predecir tendencias. Piénsese solamente que hace apenas unos pocos años fue imposible — aún para los más reconocidos especialistas - predecir los actuales desarrollos de la digitalidad y del internet.

Los análisis realizados por mí para el IESALC muestran que la «educación virtual» de la región se encuentra aún en las primeras etapas del proceso de digitalización de la educación, y que Colombia se encuentra en niveles intermedios de desarrollo. Esos son los hechos concretos.

Con todo, basado en mis trabajos y en los datos de los estudios nacionales realizados para el IESALC, Francisco Maella desarrolló un índice que busca indagar la capacidad de los países para continuar avanzando en educación virtual. Esta información muestra que a pesar que otros países como Chile, Uruguay, Venezuela y Costa Rica disponen de mejores condiciones en algunas variables del alistamiento digital, Colombia (junto con Brasil, México y Argentina) estaría en mejor capacidad y demanda para desarrollos futuros (ver Cuadro 4), lo que es dato muy promisorio. 
Gráfico 3. Segmentación de los países según etapas de desarrollo de la educación virtual

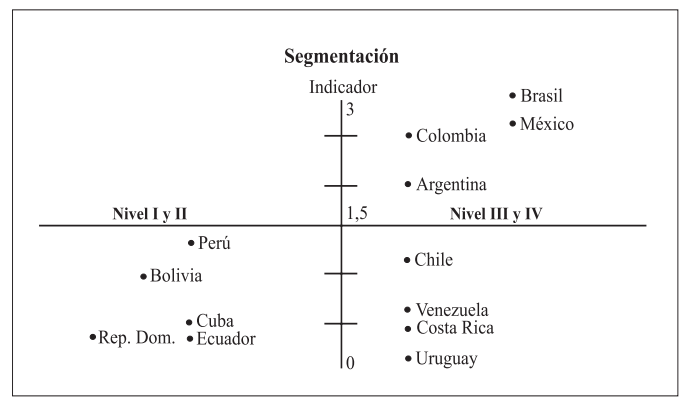

Nota. Información extraída de los estudios nacionales de UNESCO/IESALC

Elaboración: F. Maella, Penta Plus.

Yo no me atrevo a aventurar predicciones. En el desarrollo de la «educación virtual» intervienen muchos factores difíciles de conocer y controlar. Ustedes sacarán sus propias conclusiones sobre los avances, posibilidades e impactos de la educación virtual.

Es de todos nosotros conocido que, en la «sociedad del conocimiento» (que en muchos países apenas se avizora) la educación potenciada con las tecnologías digitales ofrece ya, y de forma concreta, posibilidades inmensas para el desarrollo económico y social, así como para el éxito individual, en los cambiantes y cada vez más exigentes mercados de trabajo. Como tal, es un lugar común afirmar que se constituyen en un factor de desarrollo.

Con todo, la sola introducción de las tecnologías digitales no basta para obtener los posibles y esperados impactos sociales positivos. Las características humanas, infraestructurales, pedagógicas y organizacionales (algunos de cuyos elementos he tratado de señalar) son elementos claves intervinientes y asociados en la obtención de los logros. En nuestras manos está comprenderlos y superar los condicionamientos negativos, para poder continuar avanzando de forma más dinámica y eficaz.

Lo que sí me atrevo a afirmar, a manera de conclusión, es que en algún momento hemos de tomar la decisión de cambiar el ciclo del conocimiento para que dichos impactos se den. Es decir, atrevernos a superar la actual dependencia cognoscitiva y tecnológica, produciendo aquí y ahora el conocimiento y las aplicaciones requeridas por nuestra sociedad.

La mejor estrategia al respecto es consolidar, lo antes posible, una adecuada «gestión de conocimiento» que en nuestro país sigue siendo una asignatura pendiente o al menos con desarrollos incipientes. Para ello se hace necesario investigar sobre la evolución de esta nueva área interdisciplinaria de conocimiento y de acción, sus conceptualizaciones e interrelaciones con otras áreas, sus campos de investigación y aplicación; formar equipos de investigadores en la materia; y crear una conciencia ciudadana, de tal forma que se puedan identificar y acometer programas y líneas de desarrollo y de intervención por medio de políticas de estado, así como de acciones mancomunadas y estables entre el sector educativo y el sector privado. Los impactos sociales positivos no se producen por generación espontánea. 
ANEXO A. Cuadro 2

\section{LATINOAMÉRICA Y EL CARIBE RESULTADOS DEL ÍNDICE DE ACCESO DIGITAL (DAI) (Resumen)}

\begin{tabular}{|c|c|c|c|c|c|c|c|c|c|c|c|c|c|c|}
\hline & Economía & $\begin{array}{c}\text { Suscript. } \\
\text { de lineas } \\
\text { por } 100 \\
\text { hab }\end{array}$ & $\begin{array}{c}\text { Suscript. } \\
\text { celulares } \\
\text { por } 100 \\
\text { hab }\end{array}$ & $\begin{array}{c}\text { Internet } \\
\text { tarifa } \\
\text { como } \% \\
\text { del PIB }\end{array}$ & $\begin{array}{c}\text { Analfa } \\
\text { betismo } \\
\text { adulto }\end{array}$ & $\begin{array}{l}\text { Matrícula } \\
\text { Escolar }\end{array}$ & $\begin{array}{c}\text { Banda } \\
\text { ancha } \\
\text { de } \\
\text { internet } \\
\text { Inter. } \\
\text { por } 100 \\
\text { hab }\end{array}$ & $\begin{array}{c}\text { Suscri } \\
\text { pt. } \\
\text { banda } \\
\text { ancha } \\
\text { por } \\
100 \\
\text { hab }\end{array}$ & $\begin{array}{c}\text { Usuarios } \\
\text { internet } \\
\text { por } 100 \\
\text { hab. }\end{array}$ & $\begin{array}{l}\text { Infraes- } \\
\text { tructura }\end{array}$ & $\begin{array}{l}\text { Capaci } \\
\text { dad de } \\
\text { compra }\end{array}$ & $\begin{array}{l}\text { Conoci- } \\
\text { miento }\end{array}$ & $\begin{array}{l}\text { Calidad } \\
\text { de uso }\end{array}$ & $\begin{array}{c}\text { Indice de } \\
\text { Acceso } \\
\text { Digital } \\
\text { (DAI) }\end{array}$ \\
\hline \multicolumn{15}{|l|}{ ALTO } \\
\hline Suecia & 65,2 & 88,9 & 1,1 & 98,5 & 113 & 10.611 & 8 & 57,3 & 0,94 & 0,99 & 0,99 & 0,64 & 0,67 & 0,85 \\
\hline
\end{tabular}

\begin{tabular}{|c|c|c|c|c|c|c|c|c|c|c|c|c|c|c|}
\hline \multicolumn{15}{|l|}{ SUPERIOR } \\
\hline Bahamas & 40,6 & 39 & 2 & 95,5 & 74 & 464,7 & 6,3 & 19,2 & 0,53 & 0,98 & 0,88 & 0,49 & 0,23 & 0,62 \\
\hline $\begin{array}{l}\text { St. Kitts } \\
\text { and Neyjs }\end{array}$ & 50 & 31,9 & 4,2 & 97,8 & 70 & 42,2 & 1,1 & 21,3 & 0,58 & 0,96 & 0,89 & 0,32 & 0,25 & 0,6 \\
\hline Chile & 23 & 42,8 & 6,1 & 95,9 & 76 & 131,6 & 1,3 & 23,8 & 0,41 & 0,94 & 0,89 & 0,36 & 0,28 & 0,58 \\
\hline $\begin{array}{l}\text { Antigua \& } \\
\text { Barbuda }\end{array}$ & 47,8 & 32,1 & 2,8 & 86,6 & 69 & 359 & 0 & 12,8 & 0,56 & 0,97 & 0,81 & 0,38 & 0,15 & 0,57 \\
\hline Barbados & 47,9 & 19,7 & 3,2 & 99,7 & 89 & 24,2 & 0 & 11,2 & 0,5 & 0,97 & 0,96 & 0,28 & 0,13 & 0,57 \\
\hline Uruguay & 28 & 19,3 & 7,3 & 97,6 & 84 & 128,5 & 0 & 13,6 & 0,33 & 0,93 & 0,93 & 0,34 & 0,16 & 0,54 \\
\hline Dominica & 33,3 & 13,1 & 6,3 & 96,4 & 65 & 70,2 & 0,8 & 17,5 & 0,34 & 0,94 & 0,86 & 0,33 & 0,21 & 0,54 \\
\hline Argentina & 21,9 & 17,8 & 3,9 & 96,9 & 89 & 149,6 & 0,3 & 11,2 & 0,27 & 0,96 & 0,94 & 0,35 & 0,13 & 0,53 \\
\hline $\begin{array}{l}\text { Trinidad \& } \\
\text { Tobago }\end{array}$ & 25 & 27,8 & 2,5 & 98,4 & 67 & 73,8 & 0 & 10,6 & 0,35 & 0,98 & 0,88 & 0,32 & 0,12 & 0,53 \\
\hline Jamaica & 17,2 & 53,5 & 16,9 & 87,3 & 74 & 28 & 1 & 22,9 & 0,41 & 0,83 & 0,83 & 0,3 & 0,27 & 0,53 \\
\hline Costa Rica & 25,1 & 11,1 & 7,6 & 95,7 & 66 & 114,7 & 0 & 19,3 & 0,26 & 0,92 & 0,86 & 0,34 & 0,23 & 0,52 \\
\hline St. Lucía & 32 & 8,9 & 6,9 & 90,2 & 82 & 93,8 & 0 & 11,3 & 0,31 & 0,93 & 0,87 & 0,33 & 0,13 & 0,52 \\
\hline Grenada & 31,6 & 7,1 & 7,6 & 94,4 & 63 & 37,7 & 0,5 & 14,2 & 0,3 & 0,92 & 0,84 & 0,31 & 0,17 & 0,51 \\
\hline México & 14,6 & 25,3 & 4,6 & 91,4 & 74 & 56,9 & 0,2 & 9,8 & 0,25 & 0,95 & 0,86 & 0,32 & 0,12 & 0,5 \\
\hline Brasil & 22,3 & 20,1 & 11,8 & 87,3 & 95 & 53,7 & 0,4 & 8,2 & 0,29 & 0,88 & 0,9 & 0,32 & 0,1 & 0,5 \\
\hline
\end{tabular}




\begin{tabular}{|c|c|c|c|c|c|c|c|c|c|c|c|c|c|c|}
\hline \multicolumn{15}{|l|}{ MEDIO } \\
\hline Panamá & 12,4 & 19,2 & 10,7 & 92,1 & 75 & 210,1 & 0 & 4,1 & 0,2 & 0,89 & 0,86 & 0,36 & 0,05 & 0,47 \\
\hline Venezuela & 11,2 & 25,5 & 5,7 & 92,8 & 68 & 27,3 & 0,3 & 5 & 0,22 & 0,94 & 0,85 & 0,29 & 0,06 & 0,47 \\
\hline Belice & 12,4 & 20,4 & 23,1 & 93,4 & 76 & 181,8 & 0 & 11,9 & 0,21 & 0,77 & 0,88 & 0,36 & 0,14 & 0,47 \\
\hline St. Vicente & 23,4 & 8,5 & 9,5 & 88,9 & 58 & 34,2 & 0,9 & 6 & 0,24 & 0,91 & 0,79 & 0,31 & 0,07 & 0,46 \\
\hline Suriname & 16,5 & 22,8 & 18,5 & 94 & 77 & 25,2 & 0 & 4,2 & 0,25 & 0,82 & 0,88 & 0,28 & 0,05 & 0,46 \\
\hline Colombia & 17,4 & 10,6 & 12,2 & 91,9 & 71 & 12,7 & 0,1 & 4,6 & 0,2 & 0,88 & 0,85 & 0,26 & 0,05 & 0,45 \\
\hline Perú & 7,6 & 8,6 & 19,2 & 90,2 & 83 & 45,6 & 0,1 & 9,3 & 0,11 & 0,81 & 0,88 & 0,31 & 0,11 & 0,44 \\
\hline Guyana & 9,2 & 9,9 & 29,8 & 98,6 & 84 & 3,5 & 0 & 14,2 & 0,13 & 0,7 & 0,94 & 0,21 & 0,17 & 0,43 \\
\hline $\begin{array}{l}\text { Rep. } \\
\text { Dominicana }\end{array}$ & 10,4 & 19,5 & 17,1 & 84 & 74 & 5,9 & 0 & 3,4 & 0,18 & 0,83 & 0,81 & 0,23 & 0,04 & 0,42 \\
\hline Ecuador & 11,4 & 12,6 & 26,3 & 91,8 & 72 & 6,1 & 0,1 & 4,3 & 0,16 & 0,74 & 0,85 & 0,23 & 0,05 & 0,41 \\
\hline $\begin{array}{l}\text { Paraguay } \\
\end{array}$ & 4,7 & 28,8 & 37,3 & 93,5 & 64 & 17,3 & 0 & 1,7 & 0,18 & 0,63 & 0,84 & 0,27 & 0,02 & 0,39 \\
\hline Guatemala & 7,1 & 13,1 & 21,4 & 69,2 & 57 & 72,9 & 0 & 3,3 & 0,12 & 0,79 & 0,65 & 0,32 & 0,04 & 0,38 \\
\hline El Salvador & 10,3 & 13,8 & 27,8 & 79,2 & 64 & 6,7 & 0 & 4,6 & 0,15 & 0,72 & 0,74 & 0,24 & 0,05 & 0,38 \\
\hline Bolivia & 6,8 & 10,5 & 29,8 & 86 & 84 & 2,2 & 0 & 3,2 & 0,11 & 0,7 & 0,85 & 0,19 & 0,04 & 0,38 \\
\hline Cuba & 5,1 & 0,2 & 29,8 & 96,8 & 76 & 4,6 & 0 & 1,1 & 0,04 & 0,7 & 0,9 & 0,22 & 0,01 & 0,38 \\
\hline
\end{tabular}

\begin{tabular}{|l|r|r|r|r|r|r|r|r|r|r|r|r|r|r|r|}
\hline \multicolumn{10}{|l|}{ BAJO } \\
\hline Honduras & 4,8 & 4,9 & 52,9 & 75,6 & 62 & 1,5 & 0 & 205 & 0,06 & 0,47 & 0,71 & 0,18 & 0,03 & 0,29 \\
\hline Nicaragua & 3,2 & 3,8 & 138,6 & 66,8 & 65 & 6 & 0 & 1,7 & 0,05 & 0 & 0,66 & 0,23 & 0,02 & 0,19 \\
\hline Haití & 1,6 & 1,7 & 354,5 & 50,8 & 52 & 4,2 & 0 & 0,1 & 0,02 & 0 & 0,51 & 0,22 & 0,01 & 0,15 \\
\hline
\end{tabular}

Fuente:Unión Internacional de Telecomunicaciones. Reporte Mundial del Desarrollo de las Telecomunicaciones. Indicadores de Acceso a la Sociedad del Conocimiento, 2004.

Nota: Los valores del DAI se muestran en centésimas de un punto decimal. Economías con el mismo valor DAI se ordenaron en milésimas de un punto decimal. 


\section{REFERENCIAS BIBLIOGRÁFICAS}

DANE (Departamento Administrativo Nacional de Estadística). Encuesta de Medición de las Tecnologías de la Información y las Comunicaciones. Bogotá: DANE, 2001.

FACUNDO, Ángel. Antecedentes, situación y perspectivas de la educación superior virtual en América Latina y el Caribe. En: UNESCO/IESAL. Informe sobre la Educación Superior en América Latina y El Caribe 2000-2005. La metamorfosis de la Educación Superior. Caracas: Editorial Metrópolis C. A.

FACUNDO, Ángel. La educación superior a distancia/virtual en Colombia. En: La educación a distancia en América Latina. Modelos, Tecnologías y Realidades. Marta Mena, compiladora. Buenos Aires: Editorial Stella, ICDE, UNESCO/IESALC, La Crujía ediciones, 2004. Igualmente en: ANUIES, IESALC/UNESCO. La educación superior Virtual en América Latina y el Caribe. México: Asociación Nacional de Universidades e Instituciones de Educación Superior ANUIES, 2004.

FACUNDO, Ángel. La educación superior abierta y a Distancia: necesidades para su establecimiento y desarrollo en América Latina y el Caribe. Bogotá, febrero de 2002. Publicado por El Tintero, Revista virtual del Instituto Tecnológico de Estudios Superiores de Monterrey, No. 7, noviembre de 2002. México: http:eltintero.ruv.itesm.mx/um_07/ investigacion.htm Disponible igualmente en: www.iesalc.unesco.org.ve

FACUNDO, Ángel. La educación virtual en América Latina y el Caribe: Características y tendencias. Bogotá, 2003. Disponible en: www.iesalc.unesco.org.ve, y en el portal de educación virtual de la Universidad Nacional de Colombia: http:/virtual.unal.edu.co/docs/informacion/ index.html.

FACUNDO, Ángel. Tecnologías de Información y Comunicación y Educación Superior Virtual en América Latina y el Caribe: Evolución, Características y Perspectivas. Bogotá: Los Libertadores/IESALC UNESCO, 2005. Disponible en: www.iesalc.unesco.org.ve 